\title{
IONIZATION IN ATMOSPHERES OF BROWN DWARFS AND EXTRASOLAR PLANETS. II. DUST-INDUCED COLLISIONAL IONIZATION
}

\author{
Ch. Helling ${ }^{1}$, M. JARdine ${ }^{1}$, ANd F. MoKLER ${ }^{2,3}$ \\ ${ }^{1}$ SUPA, School of Physics \& Astronomy, University of St Andrews, North Haugh, St Andrews, KY16 9SS, UK; ch80@ st-andrews.ac.uk \\ ${ }^{2}$ Max-Planck-Institut für extraterrestrische Physik (MPE), Gießenbachstr. 1, D-85748 Garching, Germany \\ ${ }^{3}$ Max-Planck-Institut für Gravitationsphysik, Callinstraße 38, 30167 Hannover, Germany \\ Received 2010 December 22; accepted 2011 May 19; published 2011 July 27
}

\begin{abstract}
Observations have shown that continuous radio emission and also sporadic $\mathrm{H} \alpha$ and $\mathrm{X}$-ray emission are prominent in singular, low-mass objects later than spectral class $\mathrm{M}$. These activity signatures are interpreted as being caused by coupling of an ionized atmosphere to the stellar magnetic field. What remains a puzzle, however, is the mechanism by which such a cool atmosphere can produce the necessary level of ionization. At these low temperatures, thermal gas processes are insufficient, but the formation of clouds sets in. Cloud particles can act as seeds for electron avalanches in streamers that ionize the ambient gas, and can lead to lightning and indirectly to magnetic field coupling, a combination of processes also expected for protoplanetary disks. However, the precondition is that the cloud particles are charged. We use results from DRIFT-PHOENIX model atmospheres to investigate collisional processes that can lead to the ionization of dust grains inside clouds. We show that ionization by turbulence-induced dust-dust collisions is the most efficient kinetic process. The efficiency is highest in the inner cloud where particles grow quickly and, hence, the dust-to-gas ratio is high. Dust-dust collisions alone are not sufficient to improve the magnetic coupling of the atmosphere inside the cloud layers, but the charges supplied either on grains or within the gas phase as separated electrons can trigger secondary nonlinear processes. Cosmic rays are likely to increase the global level of ionization, but their influence decreases if a strong, large-scale magnetic field is present as on brown dwarfs. We suggest that although thermal gas ionization declines in objects across the fully convective boundary, dust charging by collisional processes can play an important role in the lowest mass objects. The onset of atmospheric dust may therefore correlate with the anomalous X-ray and radio emission in atmospheres that are cool, but charged more than expected by pure thermal ionization.
\end{abstract}

Key words: brown dwarfs - plasmas - planets and satellites: atmospheres - stars: atmospheres

Online-only material: color figures

\section{INTRODUCTION}

Both brown dwarfs and planets form clouds in their atmospheres. The radial extent of these clouds is mainly a function of gravity (Helling et al. 2011). High altitude clouds in the giant gas planet HD 189733b were inferred from transit measurements (Pont et al. 2008; Sing et al. 2009). HD 189733b was the first exoplanet to be detected in polarized light which is likely to be caused by Rayleigh scattering on submicron grains (Berdyugina et al. 2011). Lecavelier Des Etangs et al. (2008), too, suggest submicron dust grains as source of Rayleigh scattering in their analysis of the HD 189733b transit spectrum. The understanding that clouds are present in brown dwarf atmospheres was triggered by the disappearance of metal-oxide band (like TiO and $\mathrm{VO}$ ) with decreasing effective temperature (see Kirkpatrick 2005). The comparison with model atmospheres showed that cloudless models completely fail to explain the NIR colors and L-dwarf spectra (Chabrier et al. 2000; Burrows et al. 2006; Saumon \& Marley 2008). By today, various approximations of clouds as opacity source are in application (e.g., Burgasser et al. 2010; Currie et al. 2011) as the existence of clouds in brown dwarfs is well accepted. Photometric variability in L- and in T-dwarfs has been attributed to cloud evolution (e.g., BailerJones 2008; Artigau et al. 2009), a finding which is supported by our work on turbulent dust formation (Helling et al. 2001, 2004). Goldman (2008), however, could not confirm spectroscopically the so-called cloud-braking across the L-T transition based on their model atmosphere interpolation.
Scholz \& Eislöffel (2004) argue that very low mass objects $\left(0.08 \ldots 0.25 M_{\odot}\right)$ show co-rotating, magnetically induced spots, which would indicate the presence of localized magnetic field concentrations. Objects at the boundary between M-dwarfs and brown dwarfs $\left(0.08 \ldots 0.09 M_{\odot}\right)$ were studied by several groups considering the activity change across the substellar border. The presence of X-ray and $\mathrm{H} \alpha$ emission (Berger et al. 2008; Reiners \& Basri 2008) argues for a magnetically heated corona, but the associated increase in radio emission with decreasing mass suggests that the processes that are energizing such a corona differ from those on higher-mass stellar objects (Hallinan et al. 2006, 2008; Antonova et al. 2008). In this case, the solar paradigm for coronal heating, which involves the buildup and release of magnetic stresses in the corona, may be ineffective.

We have argued in Helling et al. (2011) that in the atmospheres of brown dwarfs and exoplanets, rapid ionization events can occur, even in a cloud that is globally neutral and obeys dust-gas charge equilibrium. This can only happen, however, if the grains within the dust cloud are ionized. As one possible process, electron avalanche processes in the electric field of charged grains then lead to the formation of a streamer resulting in $10^{13} \ldots 10^{14} \mathrm{~cm}^{-3}$ free charges per initial electron (Dowds et al. 2003; Li et al. 2007). Streamers are a growing ionization front that propagate into non-ionized matter and are present in lightning ladders and sprites.

The timescale on which these charges could recombine (the Coulomb recombination timescale) is longer than the time to 


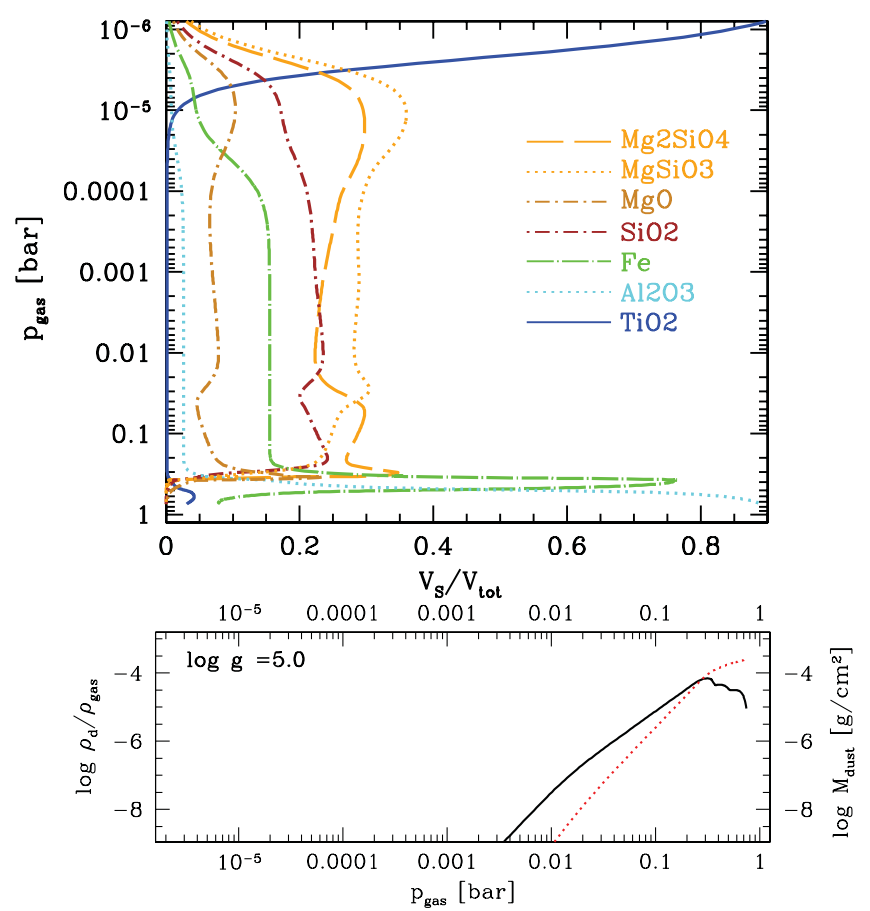

Figure 1. Material composition in volume fractions $V_{\mathrm{s}} / V_{\text {tot }}\left(V_{\mathrm{s}}\right.$-volume of a solid s, $V_{\text {tot }}$-total grain volume) of the dust cloud grains at a gas pressure $p_{\text {gas }}$ in the atmosphere (top). The bottom panel shows the dust-to-gas ratio $\rho_{\mathrm{d}} / \rho_{\mathrm{gas}}$ (solid line) and the dust mass column density $M_{\text {dust }}\left(\mathrm{g} \mathrm{cm}^{-2}\right)$ (dotted line). All quantities are results of the hydrostatic DRIFT-PHOENIX atmosphere simulation for $T_{\text {eff }}=1600 \mathrm{~K}, \log (g)=5.0$ and solar metallicity. The gas pressure $p_{\text {gas }}$ is therefore a measure for atmospheric altitude (compare, e.g., top panels in Figure 2 in Witte et al. 2009).

(A color version of this figure is available in the online journal.)

establish a streamer over a large fraction of the cloud (Helling et al. 2011). Hence, a considerable degree of ionization builds up in this time window during which the electrons could couple to the large-scale magnetic field.

In this paper, we evaluate the underlying assumption in this scenario - that the grains in substellar clouds are ionized. We concentrate on the influence of dust dynamics (sedimentation, turbulence) and kinematics (collisions) on the ionization state of brown dwarf and giant planet atmospheres. Section 2 briefly summarizes our kinetic dust cloud model and the results relevant for the topic of this paper. In Sections 3 and 4, we study the collisional energies of dust-gas and dust-dust collisions due to dust dynamics and compare these results to the ionization potential of solid compounds for an example of a brown dwarf and a gas planet atmosphere. Section 5 discusses potential lightning regimes in these atmospheres and Section 6 presents our conclusions.

\section{CLOUD FORMATION MODEL}

The formation of clouds is the formation of solid particles or liquid droplets in an atmosphere whose stratification is determined by gravity, radiation, and convection. Cloud formation in brown dwarfs and giant gas planets starts with the formation of seed particles (nucleation). This differs from the cloud formation process on Earth (or solid planets in general) where dust grains that are carried into the atmosphere from the planet's surface act as condensation nuclei. In fully gaseous objects such as brown dwarfs or giant planets those nuclei need to be built within the atmosphere itself. Once these seeds have formed, many materials are thermally stable and grow a grain mantle by
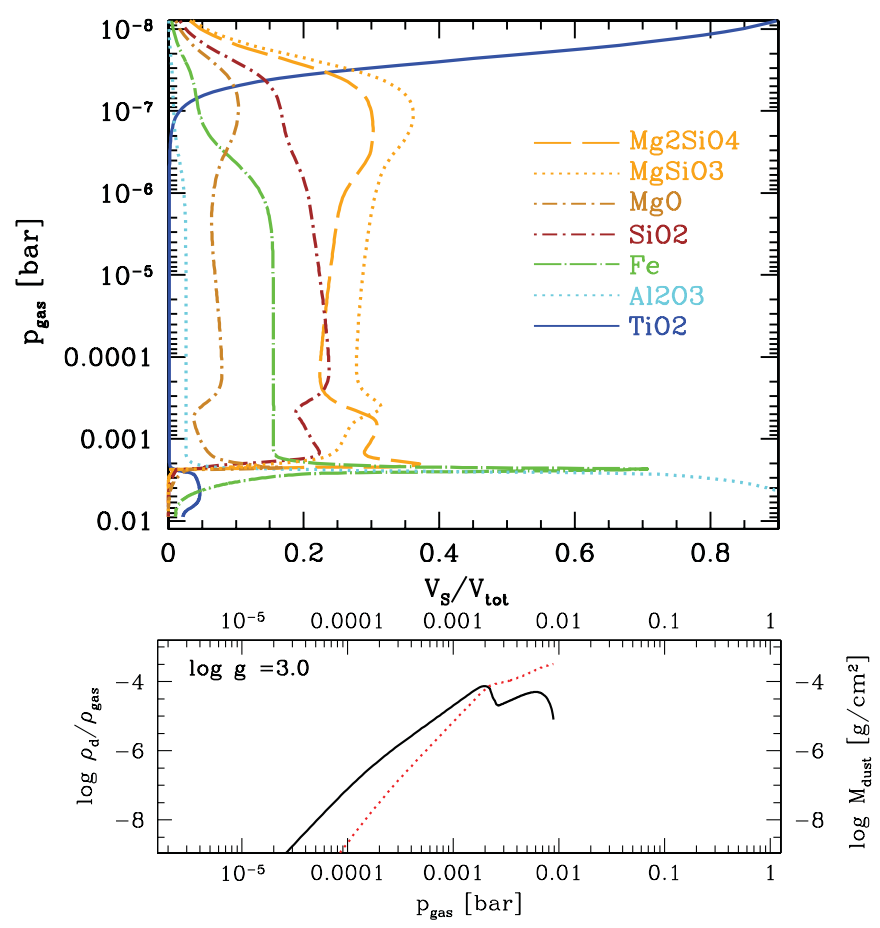

Figure 2. Same as Figure 1, but for an atmosphere simulation for $T_{\mathrm{eff}}=1600 \mathrm{~K}$, $\log (g)=3.0$, and solar metallicity.

(A color version of this figure is available in the online journal.)

chemical surface reactions on the surface of each seed particle. These compounds form patches of different, adjacent materials on the grain surfaces as different solids can grow simultaneously. Hence, these clouds are made of grains that are composed of a mix of materials that depends on the local temperature and pressure (Figures 1 and 2, top panels). As a consequence of these growth reactions, the mean particle size, $\langle a\rangle$ (Figure 3, top panels), increases.

Woitke \& Helling (2003) and Helling \& Woitke (2006) developed a model describing such heterogeneous dust formation by homogeneous nucleation, dirty growth, and evaporation. The formation of $\mathrm{TiO}_{2}$ seeds onto which solid silicate and oxide compounds grow is considered. Woitke \& Helling (2004) and Helling et al. (2008c) applied this model to the formation of stationary clouds in oxygen-rich ${ }^{4}$ atmospheres including gravitational settling, element depletion, and convective element replenishment. The atmosphere model DRIFT-PHOENIX (Dehn 2007; Helling et al. 2008a, 2008b; Witte et al. 2009) couples this detailed kinetic model of dust cloud formation with a radiative transfer code (Hauschildt \& Baron 1999; Baron et al. 2003). We use the output of the DRIFT-PHOENIX atmosphere simulations in this paper.

In DRIFT-PHOENIX the number of solids growing the mantle is restricted to seven $\left(\mathrm{TiO}_{2}[\mathrm{~s}], \mathrm{Al}_{2} \mathrm{O}_{3}[\mathrm{~s}], \mathrm{Fe}[\mathrm{s}], \mathrm{SiO}_{2}\right.$ [s], $\mathrm{MgO}[\mathrm{s}]$, $\left.\mathrm{MgSiO}_{3}[\mathrm{~s}], \mathrm{Mg}_{2} \mathrm{SiO}_{4}[\mathrm{~s}]\right)$ and to 32 surface reactions. DRIFTPHOENIX provides the local gas temperature $T(\mathrm{~K})$, the gas pressure $p_{\text {gas }}\left(\right.$ dyn $\left.\mathrm{cm}^{-2}\right)$, the maximum convective velocity $v_{\text {conv }}^{\max }$ $\left(\mathrm{cm} \mathrm{s}^{-1}\right)$, and dust quantities such as the number density of dust particles $n_{\mathrm{d}}\left(\mathrm{cm}^{-3}\right)$ of mean grain size $\langle a\rangle(\mathrm{cm})$ at each layer of the atmosphere. Because of the definition of the dust moments,

\footnotetext{
4 Oxygen-rich refers to a gas that contains more oxygen then carbon, hence the gas phase is dominated by a diversity of oxygen-binding molecules compared to a carbon-rich gas. The models used here apply the solar element abundances which are oxygen-rich.
} 

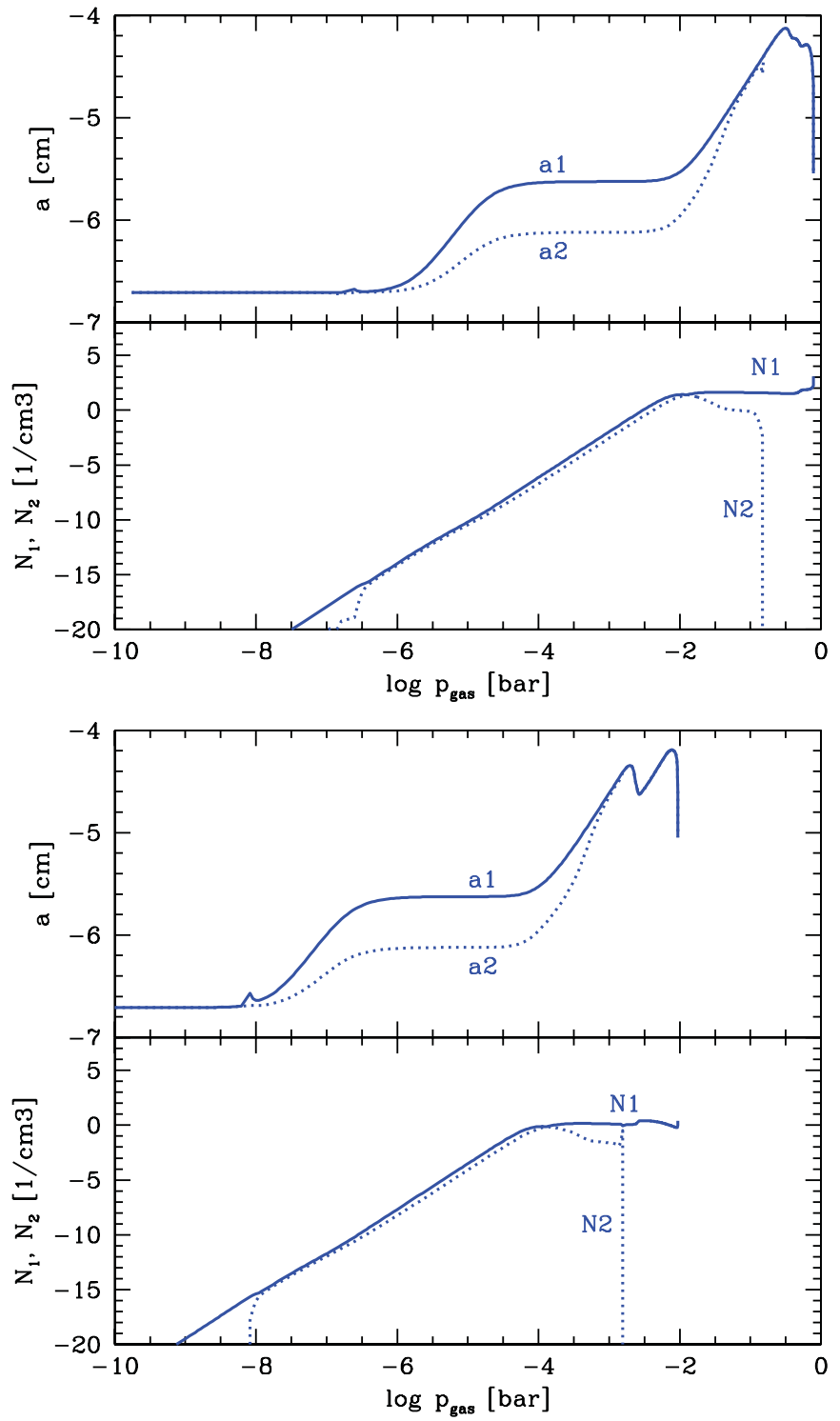

Figure 3. Height-dependent grain size distributions as result from the DRIFTPhoenix atmosphere simulation. Top panels: grain sizes $\log (a)$. Bottom panels: the number of grains $\log (N) .\left(a_{1}, N_{1}\right)$ and $\left(a_{2}, N_{2}\right)$ represent small and large particle ensemble in Equation (1). Both model atmospheres are for $T_{\text {eff }}=1600 \mathrm{~K}$ and solar metallicity. The top figure represents a brown dwarf with $\log (g)=5.0$, and the bottom figure a giant gas planet with $\log (g)=3.0$.

(A color version of this figure is available in the online journal.)

$\rho L_{\mathrm{j}}=\int_{\mathrm{V}_{\mathrm{l}}}^{\infty} V^{\mathrm{j} / 3} f(V) d V$ ( $V$-grain volume, $V_{\mathrm{l}}$-volume of smallest possible grain), the dust moments $L_{\mathrm{j}}(j=1, \ldots, 4)$ that result from a DRIFT-PHOENIX simulations are used to derive a representative grain size distribution function (for details see Appendix A in Helling et al. 2008c). We consider in this paper a double-peaked grain size distribution $f(a)$,

$$
f(a)=N_{1} \delta\left(a-a_{1}\right)+N_{2} \delta\left(a-a_{2}\right)
$$

to calculate the relative dust velocities (Sections 4.1.2 and 4.1.3) between grains of different sizes $a$ in each layer of the atmospheric cloud (Figure 3). Parameters $N_{1}, N_{2}, a_{1}$ and $a_{2}$ are determined such that the resulting dust moments reproduce the solution of the dust moment equations from the DRIFT-PHOENIX model atmosphere. Seed particles have the smallest size, $a_{1}$, of all grains in the cloud and populate the uppermost cloud layers. The cloud deck is defined as where the seed formation rate is maximum, but a small amount of very small particles also form above the cloud deck.

While the dust grains are forming and increasing in size they fall under the influence of gravity into regions of increasing density (gravitational settling, sedimentation). The increasing gas density results in faster grain growth, such that the grains increase their size considerably over short distances, and in a decreasing sedimentation velocity. The relative velocities between dust and gas due to gravity can be considered as the equilibrium drift velocity resulting from an equilibrium between gravity and friction with the surrounding gas (see Woitke \& Helling 2003). The grains fall inward (i.e., downward) and they may reach a depth where the temperature is too high to sustain their thermal stability. For the example of a brown dwarf atmosphere, at $T$ ( $p_{\text {gas }} \approx 0.4$ bar) in Figure 1 (top panel), the silicates evaporate, and instead, iron takes up $\approx 75 \%$ of the grain volume. At even higher temperatures, i.e., higher gas pressures in Figure 1 (top panel), the dust evaporates completely which causes the decrease in grain size (Figure 3). The same behavior is found for the example of a giant planet atmosphere but at lower pressure of $p_{\text {gas }} \approx 0.0025$ bar. In both cases, the dust-togas ratios, $\rho_{\mathrm{d}} / \rho_{\text {gas }}$ (black solid line, bottom, Figures 1 and 2), are highest just above the evaporation zone where also most of the dust mass per $\mathrm{cm}^{2}$ (red dotted line) is located.

The dust influences the convective velocity $v_{\text {conv }}$ indirectly through the "backwarming" effect of its opacity on the local temperature gradient of the atmosphere. The backwarming changes the convective behavior of the atmospheres because of a flatter or steeper local temperature gradient which then can suppress $\left(\delta T / \delta r<\delta T /\left.\delta r\right|_{\text {adiabatic }}\right)$ or initiate $\left(\delta T / \delta r>\delta T /\left.\delta r\right|_{\text {adiabatic }}\right)$ convection locally resulting in detached convection layers. The observation of this effect is not new in model atmospheres and it can already be found in the first papers in brown dwarf atmosphere modeling (e.g., Tsuji 2002; Burrows et al. 2006). The convective energy transport in the DRIFT-PHOENIX atmosphere simulations is considered in the mixing-length approximation from which we derive a large-scale convective velocity $v_{\text {conv }}$. Helling (2005) showed that dust formation also influences the local velocity field by radiative cooling again due to the dust's large opacity. Hence, dust formation can sustain turbulence which enhances the relative velocities of dust grains on smaller scales than convection. However, the influence of the dust on the local turbulence spectrum is not taken into account in the DRIFT-PHOENIX atmosphere simulation.

In this paper, we use local quantities which have been self-consistently evaluated in the DRIFT-PHOENIX atmosphere simulation (except for the turbulence spectrum) describing the formation of dust falling through a radiative and convective atmosphere. We investigate to what extent collisional processes are capable of ionizing dust grains in clouds in atmospheres of brown dwarfs and giant gas planets. Figure 3 shows the dispersion in grain sizes $\log (a)$ which produces a range of grain velocities at each height in the atmosphere (compare also Helling et al. 2006; Helling \& Rietmeijer 2009). Various processes may influence the relative velocities between grains (Brownian motion, convection, gravitational settling, and turbulence). We are particularly interested in whether collisional energies might then be large enough to overcome the ionization potential of an electron on the grain surface, and hence if and how these processes can contribute to charge cloud particles in the atmospheres of brown dwarfs and planets.

We note that the local $\left(T, p_{\text {gas }}\right)$ structure can differ amongst model families due to differences in cloud modeling approaches 
(Helling et al. 2008d). Even model atmosphere simulations for hotter objects differ enough to impact disk detection rates (Sinclair et al. 2010). We are, however, confident that the DRIFT-PHOENIX model atmospheres are a very reasonable attempt, as the comparison to observations in the DwarfArchieve demonstrates (Witte et al. 2011).

We perform our study for a brown dwarf and a gas planet case. We choose two solar metallicity DRIFT-PHOENIX models for a rather cool atmosphere of $T_{\text {eff }}=1600 \mathrm{~K}$. The highgravity case $(\log (g)=5.0)$ serves as an example for a brown dwarf atmosphere and the low-gravity case $(\log (g)=3.0)$ is the example for a gas planet atmosphere. This brown dwarf atmosphere model was also used in Helling et al. (2011).

\section{WORK FUNCTIONS FOR MIXED-MATERIAL DUST}

The release of an electron from the grain's surface requires that it overcomes its ionization potential, which is the energy needed to extract an electron from a surface and transport it to infinity. We assume this to be the work function in metals or the contact potential for insulators (see discussion in Poppe \& Schräpler 2005). The values of the work function are mostly given for pure atomic materials in the literature (Desch \& Cuzzi 2000; Kopnin et al. 2004) and range from $\approx 2$. . 6 eV (Figure 5). However, our grains are made of well-mixed solid materials forming from an oxygen-rich gaseous environment, and they are only partially dielectric. Rosenberger (2001) states that the work function of multi-material grains is typically lower than the ionization potential of pure atomic materials. We therefore conclude that the work function for grains of mixed materials is not well constrained, and we indicate the potential range of work functions in Figure 5 (orange/grey bar). The upper value may be somewhat overestimated leaving space for processes that were not taken into account. Ideally, these electrons would be free to move around, but grains are observed to preferentially produce surface charges on their collisional partners rather than free electrons. We start our investigation considering multielectron emission due to dust collisions which may remain on the grain surface or escape if their energy is large enough. We see this process as somewhat analogous to secondary electron emission where more than one electron could be released during the collision with an electron or an ion. During such collisions, backward scattering is more efficient in lattices of semi-conductor materials or insulators compared with metals, which is of interest because our cloud model predicts particles made of a mix of materials. The secondary electron emission coefficient ranges from 2.4 for $\mathrm{MgO}$ [s] to 4.6 for $\mathrm{Al}_{2} \mathrm{O}_{3}$ [s] $(\mathrm{NaCl}[\mathrm{s}]: 6)$ and is highest for mixed materials $\left(\mathrm{Ag}-\mathrm{Cs}_{2} \mathrm{O}-\mathrm{Cs}\right.$ : 8; Niedrig 1992, p. 359). We therefore adopt these secondary electron emission coefficients, i.e., the number of free electrons produced per collision, as guidance for mixed materials. We further note in analogy to the secondary electron emission that an increased collisional energy will not necessarily cause a continuously increasing number of electron releases because the impact may affect electrons deeper in the solid material which require a larger energy.

\section{COLLISIONAL IONIZATION}

We study ionization events by collisions inside the dust cloud layers that form in brown dwarfs and planetary atmospheres. We investigate limiting cases in order to study whether grains are charged in substellar clouds and hence, whether they can act as seeds for other, more powerful mechanisms like, e.g., electron avalanche processes. We compare the collisional energies to the ionization energies of the dust grain surface. Three collision mechanisms are studied.

1. Dust-gas collisions due to gravitational settling.

2. Dust-dust collisions by differential sedimentation of cloud particles.

3. Dust-dust collisions due to turbulence.

We discuss the effect of collisional processes only. During an elastic collision, the kinetic energy remains constant as the internal energy of the colliding bodies does not change. But the internal energy changes in the case of inelastic collisions, e.g., due to chemical processes, and hence, the kinetic energy of the colliding grains must change. Such inelastic collision could cause fragmentation, erosion, sticking, deformation, or evaporation (Güttler et al. 2010), in addition to the electron release discussed here. The occurrence of these processes, which are not considered in this paper, depends on the grain mass and relative velocity.

\subsection{Collisional Energy and Work Function}

The collisional energy is calculated for two-body collisions which can either be dust-gas or dust-dust collisions. This collisional energy is then the kinetic energy for these twoparticle collisions,

$$
E_{\mathrm{col}}=\frac{1}{2} m_{\mathrm{red}} v_{\mathrm{rel}}^{2}
$$

Here, $v_{\text {rel }}$ is the relative velocity between the collisional partners and $m_{\text {red }}$ is the reduced mass for a two-particle collision. Thus for dust-gas collisions ( $\mathrm{dg}$ )

$$
m_{\mathrm{red}, \mathrm{dg}}=\frac{m_{\mathrm{d}} m_{\mathrm{g}}}{m_{\mathrm{d}}+m_{\mathrm{g}}},
$$

and for dust-dust encounters $\left(\mathrm{dd}^{\prime}\right)$

$$
m_{\mathrm{red}, \mathrm{dd}}=\frac{m_{\mathrm{d}} m_{\mathrm{d}^{\prime}}}{m_{\mathrm{d}}+m_{\mathrm{d}^{\prime}}}
$$

where $m_{\mathrm{d}}=\frac{4}{3} \pi a^{3} \rho_{\mathrm{d}}$ is the mass of a dust grain, $m_{\mathrm{d}^{\prime}}$ the mass of the colliding grain, and $m_{\mathrm{g}}=\bar{\mu}$ the mean molecular weight in (amu) of the gas.

Clearly, the collisional energy depends on the relative velocity of the collision. We therefore consider the above mentioned three possible types of collisions separately in the following sections.

\subsubsection{Dust-Gas Collisions Due to Gravitational Settling}

In Section 2 we have seen that, once the dust starts forming, each grain falls through its gaseous environment under the influence of gravity and friction. The equilibrium sedimentation velocity between gravitation and frictional force during sedimentation is reached quickly, and therefore, we can apply the equilibrium drift concept (Woitke \& Helling 2003) to calculate the sedimentation velocity, $v^{\text {sed }}(a)$. The dust sedimentation velocity is relative to the gas phase and depends on the grain size $a$. We restrict this consideration to the large Knudsen number case (for a grain moving through gas) to be consistent with the quasistatic dust model applied in Witte et al. (2009) whose DRIFTPHoEnIX model atmosphere results we will use. We, however, 
distinguish between the subsonic and the supersonic case where $c_{T}$ is the local sound speed:

$$
\begin{gathered}
v^{\mathrm{sed}}(a)=\frac{\sqrt{\pi} g \rho_{\mathrm{d}} a}{2 \rho_{\mathrm{gas}} c_{T}} \quad v^{\mathrm{sed}}<c_{T} \\
v^{\mathrm{sed}}(a)=\sqrt{\frac{4 a \rho_{\mathrm{d}} g}{3 \rho_{\mathrm{gas}}}} \cdot c_{T} \quad v^{\mathrm{sed}}>c_{T} .
\end{gathered}
$$

The dust grains move with velocities higher than the sound speed in the very outer layers of the atmosphere due to the very low densities of the gas phase in these regions. A small-scale turbulent fluid field would influence the sedimentation velocity such that grains have more time and grow to larger sizes.

\subsubsection{Dust-Dust Collisions Due to Differential Sedimentation}

Differential sedimentation due to different grain sizes provides a source of collisions amongst the dust particles themselves. Applying the above representation of the grain size distribution function (Equation (1)) in each layer of the atmospheric cloud to Equations (5) and (6) allows us to calculate relative sedimentation velocities, $v_{\text {rel }}^{\text {sed }}(a)$, between grains of different sizes in each atmospheric layer caused by differential sedimentation. In order to illustrate the range of collisional velocities we calculate the relative velocities between the lower and upper grain size limits $a_{1}$ and $a_{2}$ :

$$
v_{\mathrm{rel} \mathrm{a}_{2}, \mathrm{a}_{1}}^{\mathrm{sed}}(a)=v^{\mathrm{sed}}\left(a_{1}\right)-v^{\mathrm{sed}}\left(a_{2}\right) .
$$

At high altitudes where $p_{\text {gas }}<10^{-6}$ bar, only nucleation is possible. Therefore, the variation in grain sizes is zero and all grains have the same sedimentation velocity at a given height, hence $v_{\text {rel }}^{\text {sed }}(a)=0$. This behavior is quite general as Witte et al. (2009) show in their Figure 2 for a whole grid of substellar atmosphere models. The greatest relative velocity is achieved in the region of efficient growth where the grain size differences can be largest. However, the relative velocity drops with higher pressures because the $\rho_{\text {gas }}$ dependency dominates if $a \approx$ const because $v^{\text {sed }}(a) \sim a / \rho_{\text {gas }}\left(\right.$ or $v^{\text {sed }}(a) \sim \sqrt{a / \rho_{\text {gas }}}$ for $v^{\text {sed }}>c_{T}$; Equations (5) and (6)).

\subsubsection{Enhanced Dust-Dust Collisions in a Turbulent Gas}

An additional velocity component leading to inter-dust collisions results from a turbulent fluid field. The role of turbulence in the atmospheres of substellar objects such as brown dwarfs and planets has been studied in Helling et al. (2001). Convective mixing and gravity waves are mechanisms to drive a turbulent fluid field in a substellar atmosphere that otherwise would be damped by the viscosity of the atmospheric gas. Whereas the frictional coupling between the dust and gas is important in determining the terminal velocity during sedimentation, friction can also cause these particles to couple to the turbulent component of the gas velocity. Whether the dust grains couple to the turbulent motion of the gas depends on the relation between the frictional timescale, $\tau_{f}$, and the turnover time, $\tau_{t}$, of a turbulent eddy (e.g., Völk et al. 1980). Particles of a given size accelerate to their terminal velocity over a frictional timescale $\tau_{f}$ (i.e., the grain's acceleration timescale $\tau_{\text {acc }}$ in Section 2.5 in Woitke \& Helling 2003). They can, therefore, couple to the velocity field of those turbulent eddies whose turnover time $\tau_{t}$ is larger than $\tau_{f}\left(\tau_{t}>\tau_{f}\right)$. The frictional coupling time is for the subsonic and the supersonic case (Woitke \& Helling 2003),

$$
\tau_{f}= \begin{cases}\frac{2 \sqrt{\pi} \rho_{\mathrm{d}} a}{3 \rho_{\mathrm{gas}} c_{T}} & v^{\mathrm{sed}} \ll c_{T} \\ \frac{2 a \rho_{\mathrm{d}}}{3 \rho_{\mathrm{gas}} v^{\mathrm{sed}}} & v^{\mathrm{sed}} \gg c_{T} .\end{cases}
$$

For homogeneous, isotropic turbulence the energy dissipation rate $\epsilon$ is constant for all scales (Kolmogoroff's theory), and one finds from dimensional arguments that inside the inertial range

$$
\epsilon=C_{1} \frac{u^{3}}{l}
$$

where $u$ is the fluid velocity associated with a scale $l$. The constant $C_{1}=0.7$ (Jimenez et al. 1993). We determine the locally constant energy dissipation rate $\epsilon$ for the largest scale of maximum kinetic energy assuming $l_{\max }=H_{p}(r) / 10$ (pressure scale height $H_{p}(r) \approx 10^{4} \ldots 10^{6} \mathrm{~cm}$ depending on radial distance $r$ ) and adopt the maximum vertical rms velocity, $u$ as proposed in Freytag et al. (2010, using their Equation (5) with $\log V=\log u_{\max }$ ),

$\log u_{\text {max }}(r)=\log v_{\text {conv }}^{\max }+\log r_{v}-\left(\log p_{\text {gas }}-\log p_{\text {gas }}^{\max }\right) /\left(H_{v} / H_{p}\right)$

with $v_{\text {conv }}^{\max }$ the maximum convective velocity and $p_{\text {gas }}^{\max }$ the gas pressure at this maximum velocity taken from our DRIFTPhoenix atmosphere simulation. Equation (10) parameterizes the velocity of the large scale that drives the turbulent fluid field in the atmosphere according to the local thermodynamic conditions. Such large-scale motions can be either convection or gravity waves according to Freytag et al. (2010). Freytag et al. (2010) derive parameterizations for $H_{v} / H_{p}$ (velocity scale height of the wave amplitude; their Equation (3)) and $r_{v}$ (the ratio of maximum convection energy to wave amplitude = "mixing efficiency," their Equation (4)) from their grid of two-dimensional convection models for substellar atmospheres. In this parameterization, $H_{v} / H_{p}$ and $\log r_{v}$ are constant for a given set of stellar parameters. Hence, we find $H_{v} / H_{p}=2.855$ and $\log r_{v}=-1.348$ for our cases of $T_{\text {eff }}=1600 \mathrm{~K}$ (solar metallicity) from their Equations (3) and (4). With $v_{\text {conv }}^{\max }=4.5 \cdot 10^{3} \mathrm{~cm} \mathrm{~s}^{-1}$ and $p_{\text {gas }}^{\max }=2.0 \cdot 10^{7} \mathrm{dyn}^{-2}$ (=20 bar) for $\log (g)=5,0$, and $v_{\mathrm{conv}}^{\max }=1.7 \cdot 10^{4} \mathrm{~cm} \mathrm{~s}^{-1}$ and

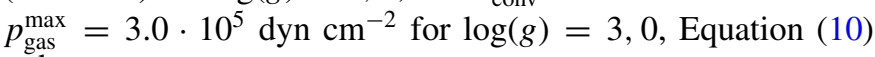
reduces to

$$
\log u_{\max }(r)=4.862-\frac{\log p_{\text {gas }}}{2.855} \quad(\text { brown dwarf, } \log (g)=5.0)
$$

$\log u_{\max }(r)=4.800-\frac{\log p_{\text {gas }}}{2.855} \quad$ (giant planet, $\log (g)=3.0$ ).

Hence, the maximum vertical rms velocity has no strong dependence on the surface gravity. The energy dissipation rate $\epsilon$ (Equation (9)) depends on the atmospheric pressure $p_{\text {gas }}$, and it therefore changes globally but it is constant in each atmospheric layer $\Delta h_{\mathrm{i}}$ (see Section 4.3).

The eddy turnover time, i.e., the correlation time of turbulent fluctuations, is

$$
\tau_{\mathrm{t}}=\frac{l}{u} \sim\left(\frac{l^{2}}{\epsilon}\right)^{1 / 3}
$$


and it depends on the locally characteristic scale $l$ (eddy size). Since $\tau_{\mathrm{t}}$ determines the lifetime of an eddy, smaller eddies have a shorter lifetime than larger eddies for a constant energy dissipation rate $\epsilon$. Hence, if we know $\epsilon$ and $l$, we can determine $\tau_{\mathrm{t}}$.

Whether and how the turbulent gas motion influences the dust dynamics depends on the relation between $\tau_{f}$ and $\tau_{t}$. Under certain conditions the turbulent gas interacts with the dust grains such that the dust grains acquire a relative inter-grain velocity component among them. In order to quantify the influence of turbulence on the dust dynamics, we use the formalism deduced by Morfill (1985) to calculate the turbulenceinduced relative (drift) velocity between the grains in the cloud, $\Delta v_{\text {ind,t }}$ :

$$
\begin{aligned}
& \Delta v_{\text {ind }, \mathrm{t}} \simeq\left\langle\delta v_{g}^{2}\right\rangle^{\frac{1}{2}}\left[\left(1+\frac{\tau_{f 1}}{\tau_{t}}\right)^{-1}+\left(1+\frac{\tau_{f 2}}{\tau_{t}}\right)^{-1}\right. \\
& \left.-2\left(\frac{1}{\left(1+\frac{\tau_{f 1}}{\tau_{t}}\right)\left(1+\frac{\tau_{f 2}}{\tau_{t}}\right)}\right)\right] \text {. }
\end{aligned}
$$

Equation (14) simplifies for grains of similar sizes $\left(\tau_{f 1} \sim \tau_{f 2}\right)$ to

$$
\Delta v_{\text {ind }, \mathrm{t}} \simeq\left\langle\delta v_{g}^{2}\right\rangle^{\frac{1}{2}} \frac{\sqrt{\frac{2 \tau_{f}}{\tau_{t}}}}{1+\frac{\tau_{f}}{\tau_{t}}} .
$$

Following Morfill (1985) we represent $\left\langle\delta v_{\mathrm{g}}^{2}\right\rangle^{\frac{1}{2}}$, which is any systematic velocity component, by Equation (10) or Equations (11) and (12). To calculate $\tau_{t}$, we consider a typical eddy scale of $l=1 \mathrm{~cm}$ to represent small-scale end of the turbulence spectrum. This choice is guided by the critical length scale $l_{\text {cri }}$ for which $\tau_{f}=\tau_{t}$ (Figure 4, top panels) i.e., when the momentum exchange between gas and dust is most efficient, and consequently the turbulence-induced inter-grain velocity component is highest. We can argue with Equation (13) that the eddies have already decayed before the grain fictionally couple to the gas $\left(\tau_{f}>\tau_{t}\right)$ for eddy sizes $l<l_{\text {cri }}$. The grains equilibrate with the gas, hence they move together with the turbulent eddies only if $\tau_{f}<\tau_{t}$, for which $l>l_{\text {cri }}$ follows. Figure 4 demonstrates that the behavior is rather diverse in the brown dwarf case for our choice of $l=1 \mathrm{~cm}$, pointing to a multi-scale problem that only very approximately can be described by one pre-chosen scale (see also Helling 2005). In the planetary atmosphere case, our choice of $l=1 \mathrm{~cm}$ suggests that all grains are frictionally coupled. If $l$ is treated in more detail, the impact of turbulent motions on dust-dust collisions will be larger in the planetary atmosphere because $l_{\text {cri }}<1 \mathrm{~cm}$. Note, however, that grains will decouple from the gas due to gravity on the largest scales in the atmosphere.

Figure 4 (bottom panels) shows the turbulence-induced relative (drift) velocity $\Delta v_{\text {ind, } t}$ for collisions of grains of different sizes (dashed line, Equation (14)) and for grains of the same size (solid and dotted lines, Equation (15)) over the whole atmospheric pressure range. The turbulence driving velocity, $u_{\max }\left(p_{\text {gas }}\right)$, is plotted in comparison. There is a maximum of $\Delta v_{\text {ind,t }}$ in both atmosphere cases, brown dwarf and gas planet, whereas $\Delta v_{\text {ind,t }}$ clearly decreases toward higher pressures. In these inner atmospheric regions, $\tau_{f}$ decreases because the gas density increases, and therefore the dust grains can adjust more quickly to the gas motion.

\subsection{Energy Release During Dust-Gas and Dust-Dust Collisions}

The collisional energies according to Equations (2), (5), (6), and Equations (7), and Equation (15) are shown in Figure 5, together with the ionization potential (work function) for a mix of solid materials as it is expected for a grain surface based on our dust formation model. We explore the results of our considerations for two solar metallicity DRIFT-PHOENIX model atmospheres: $T_{\text {eff }}=1600 \mathrm{~K}$ for $\log (g)=5.0$ (brown dwarf) and $\log (g)=3.0$ (planet).

Figure 5 shows that the collisional energies can change by 1-5 orders of magnitude throughout the cloud layer of the brown dwarf and even more within the planetary atmosphere. This change is partly directly due to the variation in grain mass which in turn depends on the local thermodynamic conditions in the atmosphere, and partly due to changing local conditions, such as the relative grain velocity, temperature, and pressure.

The collisional energies due to dust-gas collisions (not shown) are not large enough to provide the ionization energy, and hence, this kind of collisions are negligible for grain ionization. Dust-dust collisions between grains of different sizes due to differential sedimentation ("rain out," short dashed brown line) reach the ionization potential for grains only at rather low gas pressures ( $p_{\text {gas }}=10^{-5} \ldots 10^{-4}$ bar) in the brown dwarf atmosphere. Comparing Figure 5 with Figure 3 shows that this interval is related to strong changes in grain sizes due to grain growth and grain evaporation.

For turbulence-induced collisions between grains of similar sizes (green solid line, Figure 5) produce the largest collisional energies. Most of the turbulence-induced inter-grain collisions have collisional energies larger than the ionization potential and are therefore most efficient in ionizing grains inside the clouds in substellar atmospheres.

Our example of a giant gas planet atmosphere demonstrates that dust-dust collisions are only sufficient to overcome the dust material's ionization energies in the inner and densest part of the cloud, and only of turbulence amplifies the collisional process. This effect will be amplified if the inter-grain velocities are larger due to a more efficient frictional coupling than assumed in this paper. However, the geometrical height of the cloud covered is larger than in a high-gravity brown dwarf atmosphere (Figure 5).

\subsection{Electron Pressure Equivalent Due to Dust-Dust Collisions}

In the first paper of this series, we argued that charged grains can act like small capacitors and initiate electron avalanche processes that produce large numbers of free electrons. If many of these events superimpose and enough electrons leak out of the streamers, the degree of gas ionization could be increased to such an extent that the cloud might couple to a large-scale magnetic field. In this paper, we have demonstrated that the cloud particles can be charged in substellar and planetary atmospheres on the basis of turbulence-induced inter-grain collisions alone. The next step is to quantify the number of electrons that can potentially be produced by these collisions in the whole cloud. This would also allow us to investigate whether inter-grain collisions have the potential to produce enough charges to allow a coupling with a large-scale magnetic field in the case that these charges are released into the gas phase where they have a greater mobility.

The energies produced by dust-dust collisions are larger than the ionization energy by several orders of magnitude in most of the brown dwarf cloud volume or a fraction of it in gas planets if the cloud is turbulent. Such high energies, which also 

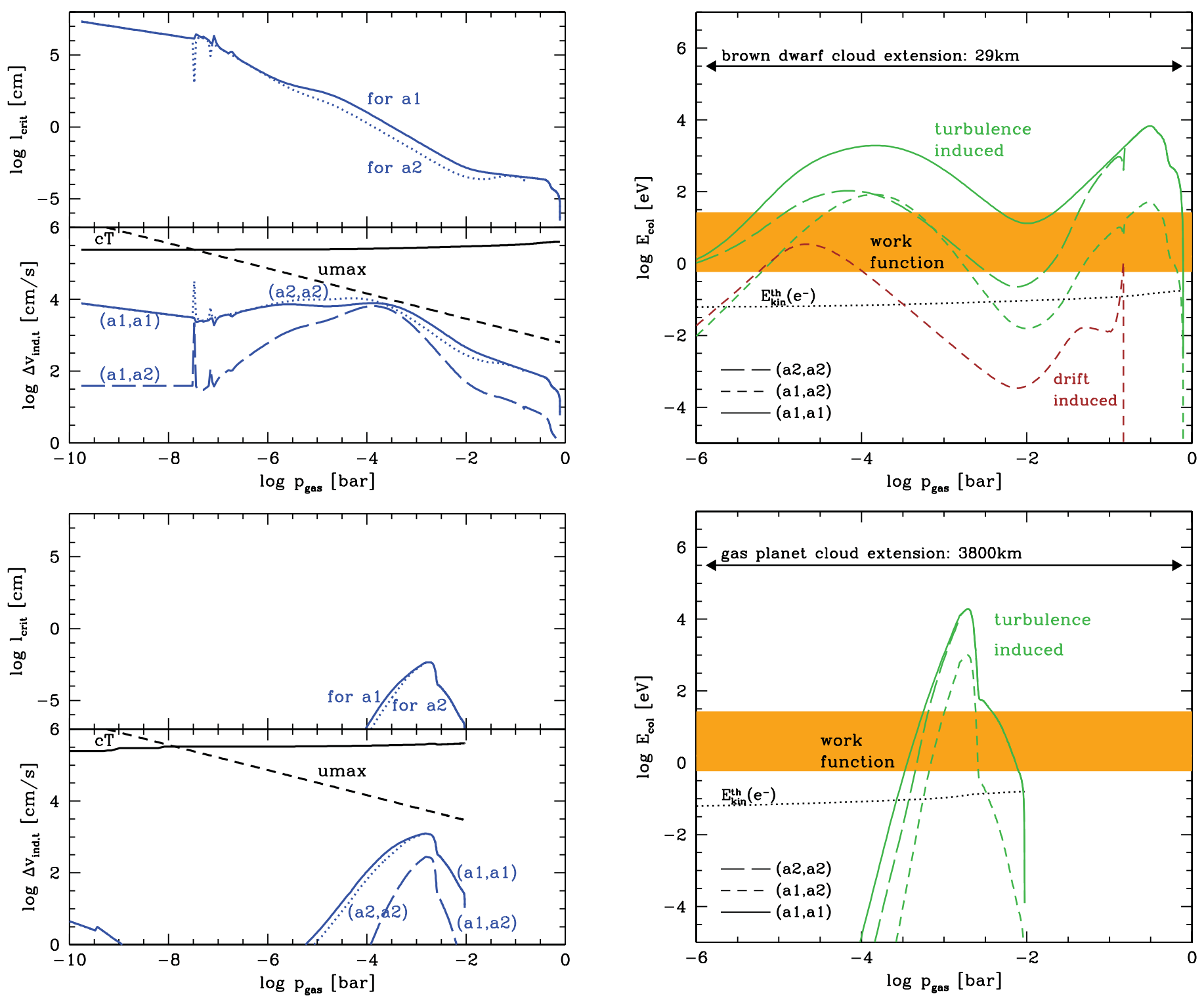

Figure 4. Top panel: critical length scale for which $\tau_{\mathrm{f}}=\tau_{\mathrm{t}}$. Bottom panel: turbulence-induced relative inter-grain velocity component $v_{\text {ind,t }}$ applying $l=1 \mathrm{~cm}$ (Equation (13)). The local sound speed $c_{\mathrm{T}}$ and the large-scale gas velocity, $u_{\max }$, which drive the turbulence in the gas are overplotted. Both model atmospheres are for $T_{\text {eff }}=1600 \mathrm{~K}$ and solar metallicity. The top figure represents a brown dwarf with $\log (g)=5.0$, and the bottom figure a giant gas planet with $\log (g)=3.0$.

(A color version of this figure is available in the online journal.)

exceed the thermal electron energy (black dotted line, Figure 5), suggest that electrons could escape from the grain surfaces into the gas. Whether the electrons affected by dust-dust collisions remain within the dust phase or whether they indeed escape from the grain surface depends on their kinetic energy gained during the collisions and on the electronegativity of the surrounding gas phase constituents. In the case of destructive grain processes, the potentially charge-carrying dust surface increases and charges could escape more easily into the gas as evaporation processes are faster. Many more mechanisms can influence this process (see introduction to Section 4) in addition to profound uncertainties in the microphysics of dust charging.

Desch \& Cuzzi (2000) consider frictional charge transfer between grains of different size. These authors assume grains of homogeneous composition, i.e., insulator grains versus metal

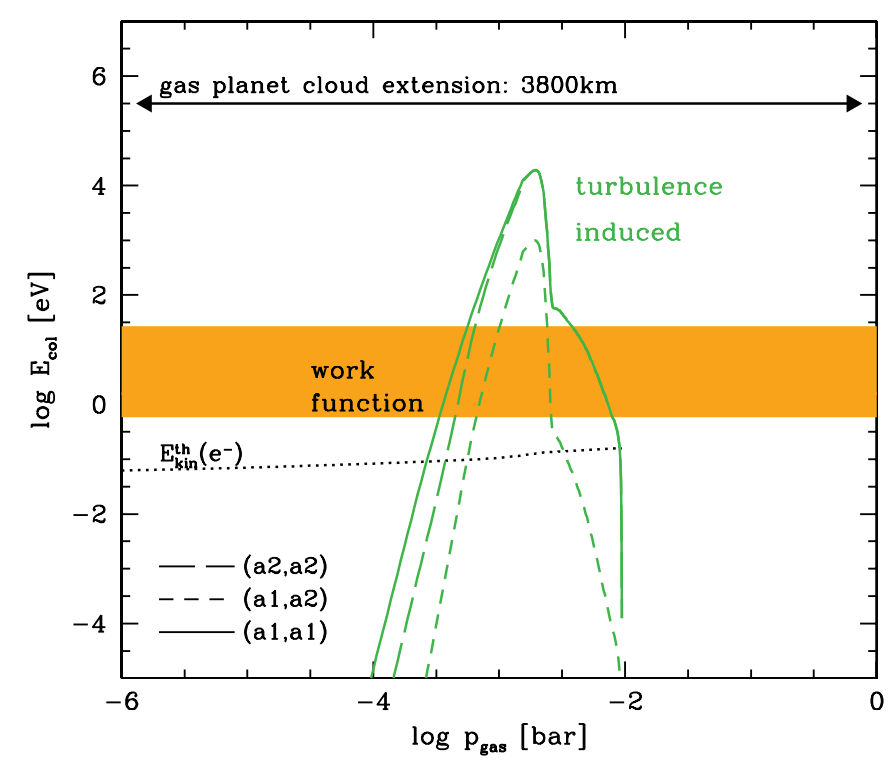

Figure 5. Collisional energies $(\mathrm{eV})$ due to turbulent dust-dust encounters (green) and due to dust-dust encounters (brown) during gravitational settling. The electrons thermal kinetic energy, $E_{\mathrm{kin}}^{\mathrm{th}}\left(e^{-}\right)$, is overplotted as black dotted line. The range of plausible dust work function is shown as orange bar. Both model atmospheres are for $T_{\text {eff }}=1600 \mathrm{~K}$ and solar metallicity. The top figure represents a brown dwarf with $\log (g)=5.0$, and the bottom figure a giant gas planet with $\log (g)=3.0$.

(A color version of this figure is available in the online journal.)

grains, which is unlikely in oxygen-rich astrophysical gases, at least in the case of substellar atmospheres. Figures 1 and 2 show that grains are composed of a mixture of metal and insulator materials over a large fraction of the cloud's extension. Hence, a charge-conservation model for homogeneous particles cannot straight forwardly be applied to substellar atmospheres. Further, the collisional energies due to turbulence-induced intergrain velocities exceed the level of ionization in some regions by a few orders of magnitude. That implies that the number of electrons that are supposed to be exchanged between dust grains by triboelectric effects according to Desch \& Cuzzi might eventually be released off the dust phase, having gained a certain amount of kinetic energy during the collision. We therefore approximate these possibly free electrons in the form of a nonthermal electron pressure to quantify the effect. 
We consider the frequency of collisions ${ }^{5}$ between dust particles of radius $a$ and dust grains of another size $a^{\prime}$. We denote the size distribution of these collisional partners as $f\left(a^{\prime}\right)$ and write the collision frequency $v_{\mathrm{col}}$ as

$$
v_{\mathrm{col}}=\pi \int_{\mathrm{a}_{\mathrm{l}}}^{\infty} a_{\mathrm{red}}^{2} v_{\mathrm{rel}}\left(a, a^{\prime}\right) f\left(a^{\prime}\right) d a^{\prime}
$$

with $a_{\text {red }}=a a^{\prime} /\left(a+a^{\prime}\right)$ the reduced grain radius and $a_{1}$ is the radius of the smallest grain possible (for details see Section 5 in Woitke \& Helling 2003). Assuming a delta-function-like representation of the grain size distribution function $f\left(a^{\prime}\right)=$ $n_{\mathrm{d}}^{\prime}\left(a^{\prime}\right) \delta\left(a-a^{\prime}\right)$, Equation (16) simplifies to

$$
v_{\mathrm{col}}=\pi a_{\mathrm{red}}^{2} v_{\mathrm{rel}}\left(a, a^{\prime}\right) n_{\mathrm{d}}^{\prime}\left(a^{\prime}\right) .
$$

Here, $n_{\mathrm{d}}\left(\mathrm{cm}^{-3}\right)$ is the number density of the collisional partner of the grain of size $a$. The relative velocity $v_{\text {rel }}\left(a, a^{\prime}\right)$ between grains of different sizes given by Equation (7), Equations (14) and (15). From Figure 5 we conclude that only Equations (14) and (15) are relevant so far.

We consider dust grains of size $a$ passing through consecutive cloud fractions of thickness $\Delta h_{\mathrm{i}}(T, p)\left(\Sigma_{\mathrm{i}} \Delta h_{\mathrm{i}}=\right.$ geometrical cloud extension $\Delta H_{\text {cloud }}$, Figure 1 in Helling et al. 2011) colliding with other grains at a certain height in the atmosphere. Note that $\Delta h_{\mathrm{i}}$ will increase with decreasing $\log (g)$ as the geometrical extension of the cloud, $\Delta H_{\text {cloud, }}$, changes with surface gravity (Figure 1 in Helling et al. 2011).

The total number of potentially released electrons per unit volume in a large-scale velocity field within each $\Delta h_{\mathrm{i}}$ is then given by

$$
n_{\mathrm{col}}^{\mathrm{tot}}=N \cdot v_{\mathrm{col}} \int_{\mathrm{a}_{\mathrm{l}}}^{\infty} f(a) \frac{\Delta h_{\mathrm{i}}}{v^{\mathrm{sed}}(a)} d a .
$$

$N$ is the number of electrons produced per collision. In case of a process of comparable efficiency such as secondary electron emission, $N \sim 8$ elementary charges would be produced per collision with a grain made of mixed materials (Section 3). For other processes, $N$ can be larger by orders of magnitude. Also smaller $\Delta h_{\mathrm{i}}$ might reveal other, small-scale velocities as leading terms compared to the large-scale velocity considered in Equations (18) potentially increasing the number of collisions $n_{\text {col }}^{\text {tot }}$.

$$
\begin{aligned}
\text { Using } f(a)= & n_{\mathrm{d}}(a) \delta(a), \text { Equation (18) simplifies to } \\
& n_{\mathrm{col}}^{\mathrm{tot}}=N \cdot v_{\mathrm{col}} n_{\mathrm{d}}(a) \frac{\Delta h_{\mathrm{i}}}{v^{\mathrm{sed}}(a)} .
\end{aligned}
$$

The sedimentation velocity $v^{\text {sed }}(a)$ of the impinging (primary) grains is approximated by the equilibrium drift velocity in Equations (5) and (6). This approximation seems well justified, however, since the time for particles to accelerate to their terminal fall speed $v^{\text {sed }}(a)$ is small (Woitke \& Helling 2003). Even if grains are stopped by a collision, the grains will continue falling under the influence of gravity and they will achieve their terminal drift velocity again quickly. This does not take turbulent mixing into account, which would increase the sedimentation time. Large-scale convective replenishment is taken into account as part of the atmosphere simulation where it impacts the dust

\footnotetext{
5 This frequency will increase or decrease depending on the charge number of the grain itself in a distance smaller then the Debye length. Diver \& Clarke (1996) and Stark et al. (2006) showed that the grain's electric field will be stronger if the grain departs from spherical symmetry.
}

formation process, consequently impacting the gravitational settling.

We assume that the dust and the gas (including thermal electrons) have the same temperature, $T_{\text {gas }}=T_{\text {dust }}$. Woitke \& Helling (2003) showed that the liberation of latent heat of condensation and the heating by friction are well balanced by radiative cooling and inelastic collisions with the gas particles for grain sizes to be expected in clouds of substellar atmospheres. We further assume that the electrons thermalize with the gas or dust, hence $T_{\mathrm{e}}=T_{\text {gas }}$. However, $T_{\mathrm{e}}=T_{\text {gas }}$ may lead to an underestimation of the electron pressure $p_{\mathrm{e}}$ as electrons tend to have higher kinetic energies than $T_{\text {gas }}$, particularly when originating from non-equilibrium processes like in streamers. For a first approximation, we retain this assumption and calculate an electron pressure equivalent,

$$
p_{\mathrm{e}, \mathrm{col}}=n_{\mathrm{col}}^{\mathrm{tot}} k T_{\mathrm{gas}} .
$$

We have shown in Section 4.2 that only turbulence-enhanced dust-dust collisions are capable of achieving appropriate relative grain-grain velocities to free electrons from the outer grain lattice (Figure 5). These grains have sizes between $\approx 0.01 \mu \mathrm{m}$ and $\approx 0.5 \mu \mathrm{m}$ (Figure 3 ). They are made of a mix of silicates $\left(\mathrm{Mg}_{2} \mathrm{SiO}_{4}[\mathrm{~s}], \mathrm{MgSiO}_{3}[\mathrm{~s}], \mathrm{SiO}_{2}[\mathrm{~s}]\right)$ which takes up $\approx 80 \%$ of the grain volume with $\approx 15 \%$ iron inclusions (Figures 1 and 2 ).

Figure 6 compares the atmospheric, thermal electron pressure $p_{\mathrm{e}}$ as result of the DRIFT-PHOENIX atmosphere simulation (solid black line) with the electron pressure $p_{\mathrm{e}, \text { col }}$ resulting from turbulence-enhanced dust-dust collisions producing $N=$ 8 charges (guided by the example of secondary electron production, dotted line), $N=10^{3}$ (dashed line, Desch \& Cuzzi 2000), and $N=10^{6}$. Hence, if the number of charges produced during the dust-dust collisions is comparable to the low efficiency of secondary electron production, the local electron pressure will not be affected in a brown dwarf atmosphere. It, however, can resupply the surface with charges, a precondition need for electron avalanche process and streamers as suggested by Helling et al. (2011). Other mechanisms are needed to increase the number of electrons released from the grain lattice to allow $p_{\mathrm{e}, \mathrm{col}} \gg p_{\mathrm{e}, \mathrm{gas}}$. Helling et al. (2011) demonstrated that a release of $N=10^{6}$ electrons per atmosphere layer would allow $50 \%$ of the cloud volume to couple to a large-scale magnetic field. We discussed electron avalanche processes as attractive possibility to achieve such a high number of free charges. In Section 5, we discuss in which atmospheric regimes superposition of avalanche processes during dust-dust collisions are likely.

In gas planet atmospheres, the situation becomes more favorable for dust-dust collisions to increase the local degree of gas ionization as the thermal gas pressure is lower than in brown dwarf atmospheres. Also the atmospheric volume affected is larger as the cloud has a larger geometrical extension than in brown dwarfs.

\section{DISCUSSION}

\subsection{Lightning versus Coronal Discharge}

We have demonstrated so far that dust-dust collisions can be energetic enough to release electrons from the cloud particle's lattice. These electrons can be expelled into the gas phase or can remain on the grain surface. In the case of an electron remaining on the grain's surface, an electric field will build up which can be strong enough to cause an avalanche process that produces an exponentially increasing number of free electrons. 

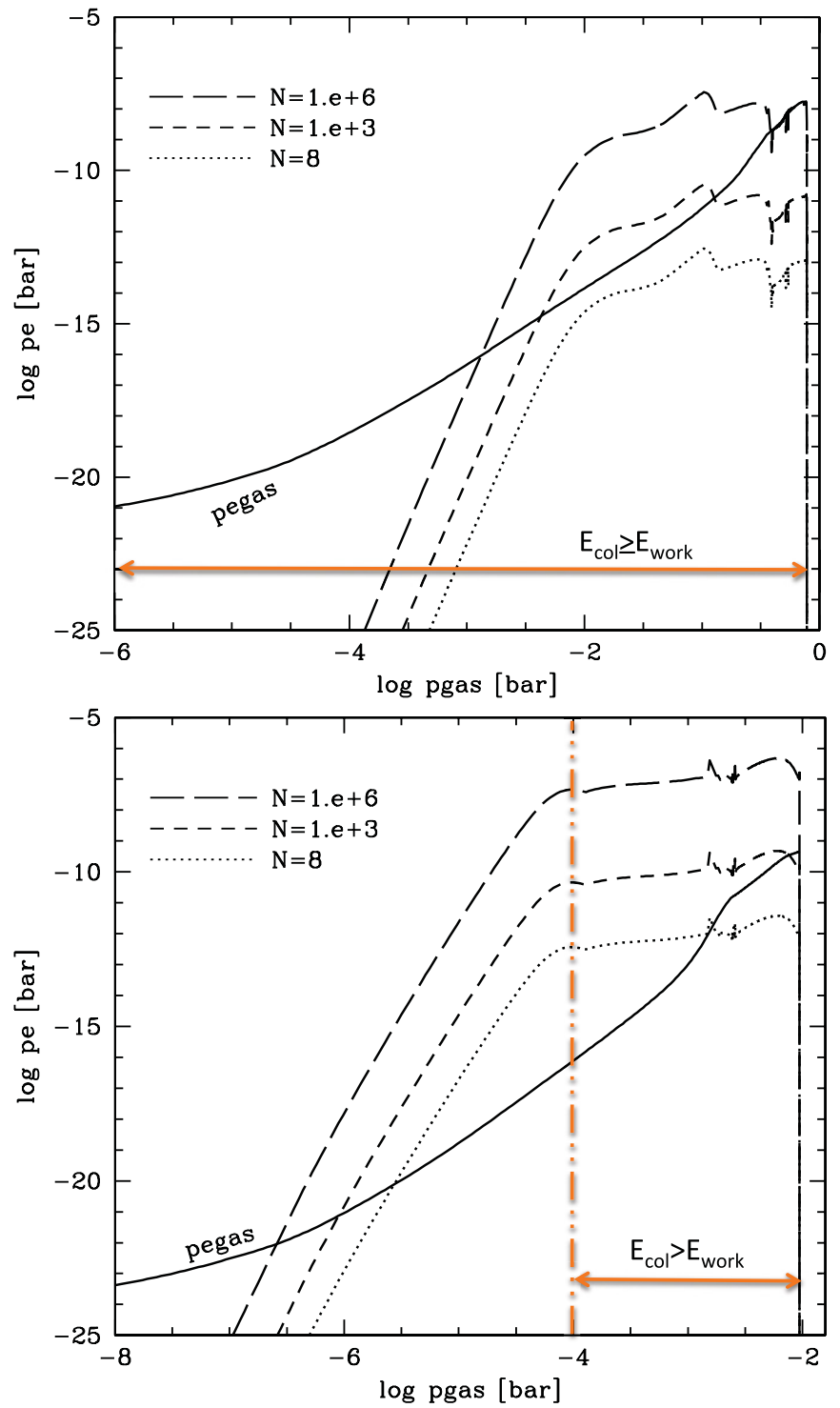

Figure 6. Electron pressure resulting from secondary electron production of $N=8$ (dotted), $N=10^{3}$ (short dashed), and $N=10^{6}$ (long dashed) electrons per dust-dust collisions compared to the atmospheric, thermal electron pressure (solid line) for two Drift-Phoenix atmosphere $\left(T_{\text {eff }}=1600 \mathrm{~K}, \log (g)\right.$ $=5.0$ (top) $/ \log (g)=3.0$ (bottom), solar metallicity). The horizontal arrow shows in which $p_{\text {gas }}$ range dust-dust collisions are energetic enough such that $E_{\text {col }}>E_{\text {work }}$.

(A color version of this figure is available in the online journal.)

As the recombination time back onto the grain surface is rather large, these electrons can exist for a time in the gas phase, and hence locally increase the degree of ionization for a certain time (Figures 6 and 7). But with which efficiency does this occur in the cloud and could it lead to the occurrence of lightning? A superposition of avalanche streamer processes will lead to more and more free electrons for a short time period which then may be defined as lightning. An estimate of the timescale, $t=n_{\mathrm{d}}^{-1 / 3} / v^{\mathrm{sed}}$, on which dust particles pass through such a previously formed electron cloud, and hence, potentially initiate another electron avalanche, shows that a certain fraction of the cloud is prone to lightning-like discharge events in brown dwarfs and in gas planets, namely, where $\tau_{\text {str }}<t<\tau_{\text {recom }}^{\text {dust }}$ in Figure 7 . The borders of this lightning region are not very clearly defined as turbulence will decrease the effective sedimentation velocity
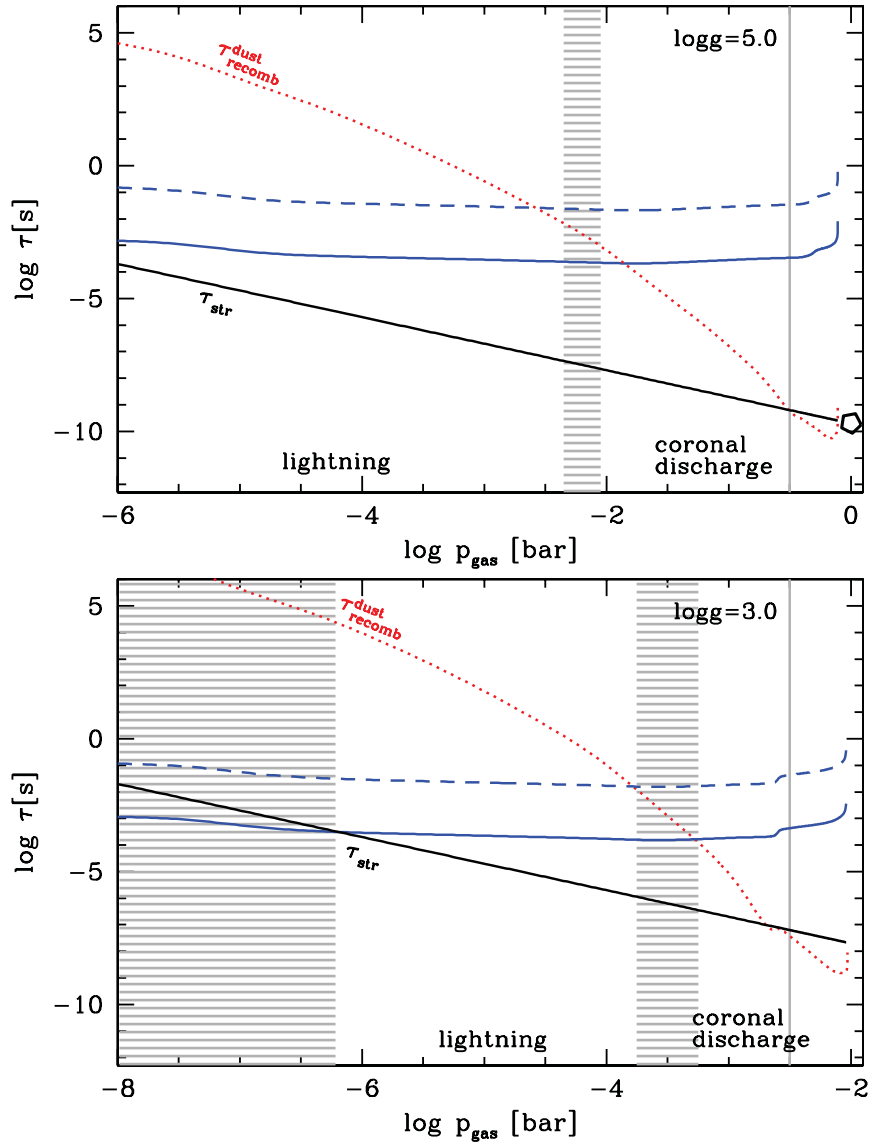

Figure 7. Timescale comparison of a streamer establishing $\left(\tau_{\mathrm{str}}\right.$, black solid line), recombination onto the dust grain ( $\tau_{\text {recom }}^{\text {dust }}$ for $q=10 \mathrm{e}$, red dotted line; Figure 4 in Helling et al. 2011), and streamer superposition timescale $t=n_{\mathrm{d}}^{-1 / 3} / v^{\text {sed }}$ (blue solid, blue dashed for $10^{2} \times v^{\text {sed }}$ ) based on the mean grain sizes from the DRIFT-PHoENIX atmospheres $\left(T_{\text {eff }}=1600 \mathrm{~K}, \log (g)=5.0 / \log (g)=3.0\right.$, solar metallicity). Multiple encounters of streamer electron clouds with the electric field of charged grains can happen in the "lightning"-regime, but not in the "coronal discharge" regime. The extension of the "lightning" regime decreases if turbulence slows down the grain settling process (blue solid line).

(A color version of this figure is available in the online journal.)

(blue solid versus dashed lines). If dust particles pass an electron cloud slowly and the electrons can recombine, lightning is less likely and a less powerful coronal discharge-like behavior on smaller scales should be expected as long as $\tau_{\text {str }}<\tau_{\text {recom }}^{\text {dust }}$.

The lightning and the coronal discharge regimes will exist in both brown dwarfs and in gas planet atmospheres. Our results suggest a hierarchy of lightning and coronal discharges with the lightning occurring at lower pressures in the upper part of the cloud. However, the maximum of the collisional dust-dust energies (Figure 5) is more likely to be located in the coronal discharge regime in our planetary atmosphere example, while dust-dust collisions act across both regimes in the brown dwarf example. How would then the cloud particles be charged if dust-dust collisions are unfavorable inside a potential lightning regime? Cosmic ray ionization may be a possibility.

Cosmic rays (CRs) seem to be an important source of atmospheric ionization in the solar system planets. Observation of Earth clouds, however, suggest that the actual charge production is not overly efficient but nevertheless important for coagulation processes. Nicoll \& Harrison (2010) determine 17 . . 150 e per particle in cloud edges on Earth which can be directly related to ionization by CRs. The charging of the cloud particles is, 
however, not a direct result of the impact of the high-energy CR particle on the cloud but rather of the ion current that develops from the CR ionization of the gas above the cloud. These ions attach to the cloud particles. The question is whether galactic CRs could be a global source of ionization for extrasolar low-mass objects.

\section{CONCLUSIONS}

Dust clouds are an integral part of the atmospheres of very low mass objects like brown dwarfs and planets. Clouds determine the local chemistry by element consumption and they influence the radiative and convective energy transport by their large opacity in the atmospheres. The aim of this paper is to demonstrate that dust grains in brown dwarf atmospheres can be charged and to investigate whether the presence of dust in brown dwarf atmospheres can contribute to its overall ionization level, a necessary condition for magnetic coupling of the atmosphere. For this purpose, we focused on collisional processes of the dust phase to cause additional ionization of the atmosphere, an aspect that had not been considered in earlier research. We, however, acknowledge that a large variety of microphysical processes can be involved into the ionization of a mineral cloud which have not yet been taken into account in our model. We find that collisional energies can be high enough to ionize the dust phase over the whole extension of atmospheric clouds of a late type brown dwarf $\left(T_{\text {eff }}=1600, \log (g)=5\right)$ and over a large part in a giant planet's atmosphere $\left(T_{\text {eff }}=1600, \log (g)=3\right)$ if the influence of the turbulent gas motion on the dust grains is taken into account. We interpret our results such that collisional ionization of the dust grains on its own does not provide, in the first place, an ionization level that is sufficiently high for magnetic coupling. A collisionally charged dust phase could, however, trigger secondary nonlinear charging processes such as electron avalanches that lead to lightning: in the case that the charging process is a charge exchange between colliding dust grains, the larger grains will be charged positively and the smaller grains would carry away the negative charges. This may lead to large-scale charge separation due to differential sedimentation and also differential response to the turbulent gas drag of grains of different mass/size.

We suggest that although thermal gas ionization may decline in objects across the fully convective boundary, dust ionization may take over in the lowest mass objects. The onset of atmospheric dust formation, the cloud depth, and its particle characteristics may therefore correlate with the anomalous $\mathrm{X}$-ray and radio emissions in atmospheres that are cool, but contain highly charged clouds.

In the context of this paper, it may be surprising that only a fraction of brown dwarfs seem to show activity in form of X-ray or radio emission and not nearly as much is known for extrasolar planets. The observed intermittency of the $\mathrm{X}$-rays might be interpreted as sign for an intermittent dust cloud distribution as suggested by us in earlier work (Helling et al. 2004). Depending on the object's parameter like mass, effective temperature, metallicity, and age, the atmosphere does change and so do the conditions for electrification which we are only beginning to explore. Our first study does suggest that cloud electrification is more pronounced in giant planets compared with the more compact brown dwarf atmospheres. However, both brown dwarfs and giant planets are prone to lightning events inside their dust clouds. Systematic investigation of this is part of our future work.
We thank the anonymous referee for a very constructive refereeing process. Ch.H. highlights the funding of the LEAP project by an FP7 ERC starting grant from the European Union, and the hospitality of the KITP Santa Barbara, University of California during the program "Theory and Observation of Exoplanets." F.M. acknowledges support by the DFG cluster of excellence "Origin and Structure of the Universe" and funding from the DFG Leibniz prize (HA 1850/28-1).

\section{REFERENCES}

Antonova, A., Doyle, J. G., Hallinan, G., Bourke, S., \& Golden, A. 2008, A\&A, 487,317

Artigau, É., Bouchard, S., Doyon, R., \& Lafrenière, D. 2009, ApJ, 701, 1534

Bailer-Jones, C. A. L. 2008, MNRAS, 384, 1145

Baron, E., et al. 2003, in IAU Symp. 210, Modelling of Stellar Atmospheres, ed. N. Piskunov, W.W. Weiss, \& D. F. Gray (Dordrecht: Kluwer), 19

Berdyugina, S. V., Berdyugin, A. V., Fluri, D. M., \& Piirola, V. 2011, ApJ, 728, 6

Berger, E., et al. 2008, ApJ, 673, 1080

Burgasser, A. J., et al. 2010, ApJ, 725, 1405

Burrows, A., Sudarsky, D., \& Hubeny, I. 2006, ApJ, 640, 1063

Chabrier, G., Baraffe, I., Allard, F., \& Hauschildt, P. 2000, ApJ, 542, 464

Currie, Th., et al. 2011, ApJ, 729, 128

Dehn, M. 2007, PhD thesis, Univ. Hamburg

Desch, S. J., \& Cuzzi, J. N. 2000, Icarus, 143, 87

Diver, D. A., \& Clarke, D. 1996, J. Phys. D: Appl. Phys., 29, 687

Dowds, B. J. P., Barrett, R. K., \& Diver, D. A. 2003, Phys. Rev. E, 68, 026412

Freytag, A., Allard, F., Ludwig, H.-G., Homeier, D., \& Steffen, M. 2010, A\&A, 513,19

Goldman, B. 2008, A\&A, 487, 277

Güttler, C., Blum, J., Zsom, A., Ormel, C. W., \& Dullemond, C. P. 2010, A\&A, 513,56

Hallinan, G., Antonova, A., Doyle, J. G., Bourke, S., Brisken, W. F., \& Golden, A. 2006, ApJ, 653, 690

Hallinan, G., Antonova, A., Doyle, J. G., Bourke, S., Lane, C., \& Golden, A. 2008, ApJ, 684, 644

Hauschildt, P. H., \& Baron, E. 1999, J. Comput. Appl. Math., 109, 41

Helling, Ch. 2005, in Interdisciplinary Aspects of Turbulence, ed. F. Kupka \& W. Hillebrandt (Garching: Max-Planck Institute), 152

Helling, Ch., Dehn, M., Woitke, P., \& Hauschildt, P. H. 2008a, ApJ, 675 , L105

Helling, Ch., Dehn, M., Woitke, P., \& Hauschildt, P. H. 2008b, ApJ, 677, L157

Helling, Ch., Jardine, M., Witte, S., \& Diver, D. A. 2011, ApJ, 727, 4

Helling, Ch., Klein, R., Woitke, P., Nowak, U., \& Sedlmayr, E. 2004, A\&A, 423,657

Helling, Ch., Oevermann, M., Lüttke, M. J. H., Klein, R., \& Sedlmayr, E. 2001, A\&A, 376, 194

Helling, Ch., \& Rietmeijer, F. J. M 2009, Int. J. Astrobiol., 8, 3

Helling, Ch., Thi, W.-F., Woitke, P., \& Fridlund, M. 2006, A\&A, 451, 9

Helling, Ch., \& Woitke, P. 2006, A\&A, 455, 325

Helling, Ch., Woitke, P., \& Thi, W.-F. 2008c, A\&A, 485, 547

Helling, Ch., et al. 2008d, MNRAS, 391, 1854

Jimenez, J., Wray, A., Saffman, P., \& Rogallo, R. 1993, J. Fluid Mech., 255, 65

Kirkpatrick, J. D. 2005, ARA\&A, 43, 195

Kopnin, S. I., Kosarev, I. N., Popel, S. I., \& Yu, M. Y. 2004, Planet. Space Sci., 52,1187

Lecavelier Des Etangs, A., Pont, F., Vidal-Madjar, A., \& Sing, O. 2008, A\&A, 481, 83

Li, C., Brok, W. J. M., Ebert, V., \& van der Mullen, J. J. A. M. 2007, J. Appl. Phys., 101, 123305

Li, E., Ebert, U., \& Brok, W. J. M. 2008, IEEE Trans. Plasma Sci., 36, 914

Morfill, G. E. 1985, in Birth and Infancy of Stars, ed. R. Lucas, A. Omont, \& R. Sorta (Amsterdam: Elsevier), 693

Nicoll, K. A., \& Harrison, R. G. 2010, Geophys. Res. Lett., 37, L31802

Niedrig, H. 1992, Physik (Berlin: Spinger)

Pont, F., Knutson, H., Gilliland, R. L., Moutou, C., \& Charbonneau, D. 2008, MNRAS, 385, 109

Poppe, T., \& Schräpler, R. 2005, A\&A, 438, 1

Reiners, A., \& Basri, G. 2008, ApJ, 684, 1390

Rosenberger, M. 2001, Astrophys. Space Sci., 277, 125

Saumon, D., \& Marley, M. S. 2008, ApJ, 689, 1327 
Scholz, A., \& Eislöffel, J. 2004, A\&A, 421, 259

Sickafoose, A. A., Colwell, J. E., Horanyi, M., \& Robertson, S. 2000, Phys. Rev. Lett., 84, 6034

Sinclair, J. A., Helling, Ch., \& Greaves, J. S. 2010, MNRAS, 409, 49

Stark, C. R., Potts, H. E., \& Diver, D. A. 2006, A\&A, 457, 365

Tsuji, T. 2002, ApJ, 575, 264
Umebayashi, T., \& Nakano, T. 2009, ApJ, 690, 69

Völk, H. J., Jones, F. C., Morfill, G. E., \& Roeser, S. 1980, A\&A, 85, 316

Witte, S., Helling, Ch., \& Hauschildt, P. H. 2009, A\&A, 506, 1367

Witte, S., Helling, Ch., \& Hauschildt, P. H. 2011, A\&A, 529, 44

Woitke, P., \& Helling, Ch. 2003, A\&A, 399, 297

Woitke, P., \& Helling, Ch. 2004, A\&A, 399, 297 